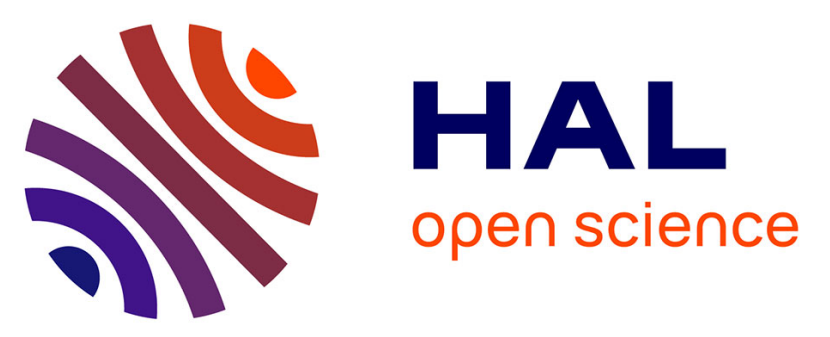

\title{
Active State of Sensory Rhodopsin II: Structural determinants for signal transfer and proton pumping
}

Ivan Yu. Gushchin, Anastasia Reshetnyak, Valentin Borshchevskiy, Andrii

Ishchenko, Ekaterina Round, Sergei Grudinin, Martin Engelhard, Georg Büldt, Valentin I. Gordeliy

\section{To cite this version:}

Ivan Yu. Gushchin, Anastasia Reshetnyak, Valentin Borshchevskiy, Andrii Ishchenko, Ekaterina Round, et al.. Active State of Sensory Rhodopsin II: Structural determinants for signal transfer and proton pumping. Journal of Molecular Biology, 2011, 412 (4), pp.591-600. 10.1016/j.jmb.2011.07.022 . hal-00766090

\section{HAL Id: hal-00766090 https://hal.inria.fr/hal-00766090}

Submitted on 17 Dec 2012

HAL is a multi-disciplinary open access archive for the deposit and dissemination of scientific research documents, whether they are published or not. The documents may come from teaching and research institutions in France or abroad, or from public or private research centers.
L'archive ouverte pluridisciplinaire HAL, est destinée au dépôt et à la diffusion de documents scientifiques de niveau recherche, publiés ou non, émanant des établissements d'enseignement et de recherche français ou étrangers, des laboratoires publics ou privés. 


\title{
Active State of Sensory Rhodopsin II: Structural determinants for signal transfer and
} proton pumping

\author{
Ivan Gushchin $^{\mathrm{ac}}$, Anastasia Reshetnyak ${ }^{\mathrm{ac}}$, Valentin Borshchevskiy ${ }^{\mathrm{ac}}$, \\ Andrii Ishchenko $^{\text {bd }}$, Ekaterina Round ${ }^{\text {ac }}$, Sergei Grudinin ${ }^{\text {ef }}$, \\ Martin Engelhard ${ }^{\mathrm{g}}$, Georg Büldt ${ }^{\mathrm{bc}}$ and Valentin Gordeliy ${ }^{\mathrm{abc} *}$
}

a Laboratoire des Protéines Membranaires, Institut de Biologie Structurale J.-P. Ebel, UMR5075 CEA-CNRS-UJF, 38027 Grenoble, France

${ }^{\mathrm{b}}$ Institute of Complex Systems (ICS), ICS-5: Molecular Biophysics, Research Centre Juelich, 52425 Juelich, Germany

c Research-educational Centre "Bionanophysics", Moscow Institute of Physics and Technology, 141700 Dolgoprudniy, Russia

${ }^{\mathrm{d}}$ Institute of Crystallography, University of Aachen (RWTH), Jaegerstrasse 17-19, 52056 Aachen, Germany

e NANO-D, INRIA Grenoble-Rhone-Alpes Research Center, 38334 Saint Ismier Cedex, Montbonnot, France

${ }^{\mathrm{f}}$ CNRS, Laboratoire Jean Kuntzmann, BP 53, Grenoble Cedex 9, France

g Max-Planck Institute of Molecular Physiology, 44227 Dortmund, Germany

* Corresponding author, tel.: +33 (0)4 387806 44; fax: +33 (0)4 387854 94; e-mail: valentin.gordeliy@ibs.fr 


\begin{abstract}
The molecular mechanism of transmembrane signal transduction is still a pertinent question in cellular biology. Generally, a receptor can transfer an external signal via its cytoplasmic surface as found for GPCRs like rhodopsin or via the membrane domain like it is utilized by sensory rhodopsin II (SRII) in complex with its transducer HtrII. In the absence of HtrII SRII functions as a proton pump. Here, we report on the crystal structure of the active state of SRII from Natronomonas pharaonis (NpSRII). The problem of a dramatic loss of the diffraction quality upon loading of the active state was overcome by growing better crystals and reducing the occupancy of the state. The determined conformational changes at the region comprising helices $\mathrm{F}$ and $\mathrm{G}$ are similar to those observed for the NpSRII-transducer complex but they are much more pronounced. Meaning of these differences for proton pumping ability and understanding of the signal transduction by NpSRII is discussed.
\end{abstract}

Key words: bacterial rhodopsins, membrane protein, active state, signaling 


\section{Introduction}

Microbial rhodopsins, a family of at least 1000 members $^{1}$, have been in the focus of recent research because of their functional diversity which encompasses ion-pumps, channels, and photosensors and because of potential applications in energy conversion, in computer memory and chip development, or in optogenetics ${ }^{2,3}$. Common features of all pigments are a seven transmembrane helix scaffold, a retinal bound to the C-terminal helix $G$ via a protonated Schiff base to a lysine residue, and a light activated photocycle. Three archetypical microbial rhodopsins can be distinguished ${ }^{2}$. Bacteriorhodopsin (BR), a proton pump, has been investigated exhaustively in great detail and is therefore a reference point for all other microbial rhodopsins. Channelrhodopsin, only recently discovered in algae, functions as a light-activated ion channel. The third system, sensory rhodopsin II (SRII) together with sensory rhodopsin I (SRI) enable archaeabacteria to seek optimal light conditions for their energy needs, while avoiding photooxidative damage. Both photoreceptors trigger a signal transduction chain which is closely related to that of the eubacterial two-component system. One essential not yet completely understood problem concerns the molecular mechanism of signal transfer from receptors to cytoplasmic signaling components. To advance the understanding of signal transduction, the crystal structure of the active state of uncomplexed sensory rhodopsin II from Natronomonas pharaonis (NpSRII) was determined.

NpSRII was chosen for these experiments because it has been characterized in quite a detail (for reviews see Klare et $a .^{2}$ and Sudo \& Spudich ${ }^{4}$ ). In the cellular membrane NpSRII is bound to its cognate transducer NpHtrII in a $2: 2$ complex $^{5,6}$ to form the signaling module. The chain of events is triggered by light thereby initiating a trans-cis isomerisation of the retinylidene chromophore of NpSRII. As a consequence the protein passes through several intermediates until it reaches back to the original state in about 1 second. The functionally 
important conformational step occurs during the $\mathrm{M} 1 \rightarrow \mathrm{M} 2$ transition which leads to the signaling state ${ }^{7,8}$. During this transition the signal is transferred through the interface comprising helices $\mathrm{F}$ and $\mathrm{G}$ to NpHtrII, which results in a rotary motion of transmembrane helix $\mathrm{TM} 2^{6,9}$. This activation occurs at the level of the membrane. How this signal is then transmitted to signal domain thereby modulating the activity of the histidine kinase CheA and triggering the cytoplasmic two component signaling cascade is not known yet ${ }^{2,5,9,10}$.

Published X-ray and NMR structures of NpSRII ${ }^{11-14}$ coincide within experimental errors and strongly resemble those obtained for bacteriorhodopsin ${ }^{15}$. The conformation of the backbone and that of conserved side-chains in the retinal-binding pocket are more or less identical. Moreover, the structure of the receptor ${ }^{11-14}$ is almost the same as compared to the NpSRII/NpHtrII complex ${ }^{5}$. These observations pose an interesting question. How does nature fine-tune common scaffolds to engender two completely different functions: sensor and ion pump? From previous work it is evident that NpSRII as well as SRI is capable of pumping protons, albeit with poor efficiency ${ }^{16-19}$. Similarly, bacteriorhodopsin can be converted into a sensor by just three mutations ${ }^{20}$. On the other hand, despite great efforts it has not been possible to change NpSRII into an efficient proton pump ${ }^{21}$. Apparently, the requirements for an effective ion pump are much more demanding than those for a functional sensor.

A second observation relates to the inhibition of the proton pump on transducer binding NpHtrII to its cognate receptor NpSRII ${ }^{22,23}$. It has been discussed that in the 2:2 complex the cytoplasmic channel cannot open sufficiently, thereby altering the proton uptake kinetics such that the reprotonation of the Schiff base is faster from the extracellular side. This kinetic correlation would result in a futile proton cycle (reviewed in Sasaki and $\mathrm{Spudich}^{24}$ ). Certainly, this proposal can only be verified by a comparison of NpSRII active states with and without bound transducer NpHtrII. 
Crystal structures are available for NpSRII ground state ${ }^{11-13}$ and for its K-intermediate, which is formed at room temperature in the nanosecond range ${ }^{25}$, as well as for the NpSRII/HtrII complex, for which data of the ground state and its $\mathrm{K}$ - and $\mathrm{M}$-intermediate (the active state) are available ${ }^{5,9}$. Despite several attempts the structures of the late (active) states of NpSRII were not obtained yet.

The reason for this failure has been related to large conformational changes upon the lightactivated M-formation, which lead to severe disturbance of the crystal packing and consequently a substantial decrease in resolution. A similar difficulties arise in experiments with the intermediate states of visual pigment rhodopsin ${ }^{26,27}$.

Here, we describe an approach which allowed us to determine the structure of an intermediate in the cases when conformational changes of a protein upon its transition from ground to an intermediate state are large and may disturb crystal packing dramatically, making crystals not suitable for X-ray crystallography. We were able to solve the structure of the active state of NpSRII. The data provide information about the movement of the functionally important helices $\mathrm{F}$ and $\mathrm{G}$. Furthermore, evidence is given about the water distribution in the cytoplasmic proton uptake channel which relates directly to the question of proton pump properties of NpSRII alone and in complex with its transducer NpHtrII. 


\section{Results}

A comparative analysis of structural changes in NpSRII alone and in NpSRII/NpHtrII necessitated crystals, grown under conditions close to those reported for the complex (as described in Gordeliy et al. ${ }^{5,9}$ and has been discussed by Reshetnyak et al. ${ }^{13}$ ). We managed to obtain the crystals at similar conditions. The addition of trehalose turned out to be not only important for obtaining high quality crystals, but was also crucial for preservation of diffraction quality during the M-state trapping.

\section{Structure of the ground state}

Using these conditions, the structure of the NpSRII ground state was obtained. The diffraction data were integrated up to $1.9 \AA$ (Table 1). The built model contains residues from 1 to 219 with poorly ordered A-B and C-D loop regions.

Similarity between the new and published X-ray structures is very high. RMSD of coordinates equals $0.31 \AA$ for $\underline{\mathbf{1 H 6 8}}^{12}, 0.43 \AA$ for $\underline{\mathbf{1 J G J}}^{11}$ and $0.27 \AA$ for the structure reported in the paper of Reshetnyak et al. ${ }^{13}$ (the position of A-B loop was omitted from calculation; RMSD was calculated for the backbone atoms). Differences are observed in the position of the C-D loop (residues 93 to 97) which in our structure is displaced by about 1.5 $\AA$ as compared to the published structures. D193 position is closer to R72, than in 1.JGJ (for $0.8 \AA$ ) as well as in $\underline{\mathbf{1 H 6 8}}$ (for $1.8 \AA$ ). We do not observe water molecules 2017 and 2026 of 1H68, but identify three additional water molecules: near the Y174 carboxyl group, near Y73 hydroxyl group and near D193. Also some small amount (occupancy < 35\%) of water molecules at position 2013 of $\underline{\mathbf{1 H 6 8}}$ may be replaced by chloride ions, as it is in $\underline{\mathbf{1 H 6 8}}$, since it would better fit the electron density. The cytoplasmic loop F-G, comprising residues 175195, is $\sim 0.5 \AA$ closer to the extracellular channel in our model. Finally, in $\underline{\mathbf{1 J G J}}$ a number of residues have a different rotameric conformation, than in our or $\underline{\mathbf{1 H 6 8}}$ structures. These 
differences may be ascribed either to different crystallization conditions and/or to a better resolution of our structure and seem not to be important for protein function.

As for the recently published NMR structure $\underline{\mathbf{2 K S Y}}^{14}$, the differences are much larger. Substantially, they include displacements of up to $1 \AA$ of residues known to be important for signal transduction (Y199, Y174 and T204), and changes in their hydrogen bonds. These differences may probably be attributed to the lack of proper restraints in NMR structure determination, the absence of water molecules and retinal proton in NMR model, or to the fact that the protein was in detergent micelles and not in the lipid bilayer.

We also observe a strong similarity between the ground-state structures of NpSRII alone and in complex with NpHtrII. RMSD of coordinates are $0.53 \AA$ for $\underline{\mathbf{1 H 2 S}}^{5}$ and $0.45 \AA$ for the structure in I212121 space group of Ishchenko et al., to be published. There are only slight differences in poorly ordered loop regions which include also the position of the C-D loop. An important observation concerns D193 which occupies the same position in the structure of the complex and the present structure, contrary to that in the structures $\underline{\mathbf{1 H 6 8}}$ and $\underline{\mathbf{1 J G J}}$. This data emphasizes the importance of identical crystallization conditions.

Additionally, it was possible to assign 31 hydrocarbon chains (Figure 1). Two of them may unambiguously be identified as archaeal phytanyl chains by their electron densities. Both eubacterial and archaeal lipids may be present in the crystal as NpSRII was expressed in E. coli and subsequentely reconstituted into $\mathrm{H}$. salinarum polar lipids. Additionally one molecule of octyl- $\beta$-D-glucopyranoside could be identified. Inclusion of the lipid molecules into the model turned out to be necessary for obtaining the active state structure of NpSRII, because it resulted in more consistent electron density maps as well as better R-factors.

\section{Heterogeneity of active state}


The procedure employed in this work did not allow trapping pure $\mathrm{M}$ state. The next intermediate in the photocycle, the O-intermediate could have been accumulated under these conditions as well. Indeed, the spectroscopic data show that the amount of the O-state reaches up to two thirds of that of the M-state (see Supplementary Information text and Supplementary Figure 1 online). Independent refinement of the $\mathrm{M}$ and $\mathrm{O}$ structures is impossible due to low data-to-parameter ratio, which is slightly over 1 when only one intermediate structure is refined. Nevertheless independent data from the literature indicate that the structure of the M-state closely resembles that of the O-state. First, the photophobic signal is relayed to the transducer in both the $\mathrm{M}$ and $\mathrm{O}$ states $^{28,29}$. EPR experiments also show that reordering of helix F and TM2 occurs, believed to represent the signal, occurs concomitantly with O-decay ${ }^{6}$. FTIR studies ${ }^{30-32}$ have shown that differences between M- and O-states are much smaller than between $\mathrm{M}$ and the ground state. Transient-grating analysis also shows little volume changes upon $\mathrm{M}$ to $\mathrm{O}$ transition compared to transition from ground to $\mathrm{M}$ intermediate ${ }^{33}$. The main difference concerns the back isomerisation of the retinal to the all-trans conformation ${ }^{30-32}$. Global similarity of the late intermediate structures was also proposed for $\mathrm{BR}^{34}$. From these considerations, it can be assumed that structures of the $\mathrm{M}$ - and O- intermediates are quite similar at present resolution. It is therefore reasonable, to treat Mand O-state as one entity. This assumption is also justified by the fact that the occupancy of the active state obtained by crystallographic means is in good agreement with the results of the spectroscopy, as $\mathrm{M}$ - and O- state combined which amounts to about 50\%. Finally, it should be noted, that in the case of the NpSRII/NpHtrII complex the trapped active state not only contained the M-intermediate but also some amounts of the O-intermediate. Nonetheless no large unaccounted difference electron densities were seen, when the data were treated with the only one model of the active state of protein. The resolution of the structure was $2.0 \AA$ 
and thus the large structural deviation in the $\mathrm{O}$ state, would it be present, would have been detected ${ }^{9}$.

\section{Trapping the active state in crystal}

Upon trapping of the active state in crystals the diffraction worsened anisotropically. The largest loss in resolution of $1 \AA$ occurs along the b-axis, which is perpendicular to the membrane plane. At the same time we observed large changes in the unit cell dimensions. The most pronounced change was again in b-axis direction, with the cell dimension

increasing from $128 \AA$ up to $131 \AA$. These effects were stronger with the higher intensity of the light used for trapping of the active state. Spectroscopy measurements showed, that illumination of the crystals (excitation wavelength $488 \mathrm{~nm}$ ) with the laser power $0.33 \mathrm{~W}$ for 2 $\mathrm{s}$ (the cryostream is blocked for that period) results in accumulation of approximately $35 \%$ $\mathrm{M}$-state and 25\% O-state. Further increase in the laser power slightly raises the amounts of the accumulated active states, to the maximum of $\sim 45 \% \mathrm{M}$-state and $30 \% \mathrm{O}$-state. These data indicate, that the more active state is present in the crystal, the larger are the diffraction resolution losses. Therefore, optimum conditions had to be established, at which both the active state occupancy and the resolution would be reasonable. We found that at the laser power $0.33 \mathrm{~W}$ the resolution loss along the b-axis does not exceed $1 \AA$, meanwhile the trapped amount of the active state allows for the structure determination. Using such an approach, a number of diffraction datasets were obtained with different NpSRII crystals. Resolution limits for the best crystal were 2.5/2.9/2.6 ̊ for a, b and c-axes correspondingly. For that crystal, amount of the active state determined crystallographically was found to be approximately $45 \%$ (see Supplementary Information text and Supplementary Figure 2 online).

\section{Conformational changes in the NpSRII active state}


As it could be predicted from the loss of diffraction quality during the trapping, we observe large conformational changes in the NpSRII active state (Figure 2A). The structural changes reported below are reproducible for 3 different illuminated crystals displaying the highest resolution, for which refinement of the active state was possible. The changes are in accordance with difference Fourier electron density maps between the active and the ground states. Thereafter we discuss the changes as they are observed in the structure of highest resolution. Alignment of structures for the comparison was done by the non-hydrogen atoms for the whole protein.

Large conformational changes are observed at the transducer-binding interface comprising helices $F$ and G. Helix F moves towards the cytoplasm by about $0.3 \AA$, whereas helix $G$ moves in opposite directions towards extracellular side by $0.7 \AA$. Helix A moves together with helix G, though with a twice as lower amplitude. The extracellular F-G loop moves out of the membrane plane. These movements are similar to those observed for the NpSRIINpHtrII complex, but display larger amplitudes (Figure 2).

Movement of the G helix, with Y199, which forms a crystal contact in case of NpSRII, seems to be the main cause for the increase of crystal b-dimension, as the increase, $3 \AA$, corresponds well to 4 times by $0.7 \AA$, which follows from packing geometry.

On the cytoplasmic surface, the E-F loop changes its conformation and becomes more compressed. The movement of the C-D loop is questionable, since this loop is poorly ordered, and its position differs as exemplified above in the known ground-state structures.

The alterations on the surface are paralleled by changes in the interior of light activated NpSRII. The difference density maps clearly show that 13 -cis retinal is present in the crystals which amounts to about $35 \%$ of the M-intermediate as determined spectroscopically (see above). Interestingly, the position of T204 relative to Y174 and helix F does not change 
(figure 3). Unfortunately, the structure resolution does not allow for the analysis of the changes in position of water molecule, bonding to W171 and T204 backbone. W171, forming a steric conflict with the retinal C13-methyl group, is pushed away from retinal by $\sim 0.6 \AA$. W76 is displaced towards helix $\mathrm{F}$ in the membrane plane.

Electron densities are observed only for few highly-ordered water molecules in the extracellular channel. Nevertheless, the absence of water molecule adjacent to the Schiff base (analogous to $\mathrm{W} 402$ of $\mathrm{BR}$ ) is clearly seen, as there are no $2 \mathrm{Fo}-\mathrm{Fc}$ densities in that region and, in addition, inclusion of this water molecule into the model results in pronounced negative difference electron density maps Fo-Fc. Disappearance of this water molecule is the result of the Schiff base deprotonation and was observed in the NpSRII/NpHtrII complex ${ }^{9}$ and in $\mathrm{BR}^{35}$ as well. 
Table 1. Crystallographics data collection and refinement statistics (molecular replacement)

\begin{tabular}{|c|c|c|}
\hline & NpSRII G-state & NpSRII active state \\
\hline \multicolumn{3}{|l|}{ Data collection } \\
\hline Space group & $\mathrm{C} 222_{1}$ & $\mathrm{C} 222_{1}$ \\
\hline \multicolumn{3}{|l|}{ Cell dimensions } \\
\hline$a, b, c(\AA)$ & $86.99,128.09,50.63$ & $86.44,131.09,51.23$ \\
\hline$\alpha, \beta, \gamma\left({ }^{\circ}\right)$ & $90,90,90$ & $90,90,90$ \\
\hline Resolution $(\AA)$ & $28-1.9(2.0-1.9)^{*}$ & $43-2.6(2.74-2.6)^{*}$ \\
\hline$R_{\text {merge }}(\%)$ & $7.0(39.3)^{*}$ & $11.7(83.7)^{*}$ \\
\hline$I / \sigma I$ & $14.4(3.8)^{*}$ & $6.5(1.5)^{*}$ \\
\hline Completeness (\%) & $96.9(97.4)^{*}$ & $92.7(86.1)^{*}$ \\
\hline Redundancy & $3.8(3.8)^{*}$ & $3.4(3.1)^{*}$ \\
\hline \multicolumn{3}{|l|}{ Refinement } \\
\hline Resolution $(\AA)$ & $22-1.9$ & $20-2.6,2.9,2.6^{* *}$ \\
\hline No. reflections & $20672(1107 * * *)$ & $7633(401 * * *)$ \\
\hline$R_{\text {work }} / R_{\text {free }}(\%)$ & $15.34 / 17.31$ & $25.12 / 27.58$ \\
\hline \multicolumn{3}{|l|}{ No. atoms } \\
\hline Protein & $1694 * * * *$ & $3184(1552 * * * * *)$ \\
\hline Ligand/ion & 375 & $726(352 * * * * *)$ \\
\hline Water & 73 & $88(15 * * * * *)$ \\
\hline \multicolumn{3}{|l|}{$B$-factors } \\
\hline Protein & 11.09 & $18.39(18.08 * * * * *)$ \\
\hline Ligand/ion & 58.70 & $54.30(54.27 * * * * *)$ \\
\hline Water & 27.28 & $22.66\left(27.15^{* * * * *}\right)$ \\
\hline \multicolumn{3}{|l|}{ R.m.s. deviations } \\
\hline Bond lengths $(\AA)$ & 0.013 & 0.012 \\
\hline Bond angles $\left({ }^{\circ}\right)$ & 1.248 & 1.393 \\
\hline
\end{tabular}

*Values in parentheses are for highest-resolution shell.

** For M-state structure anisotropic resolution limits were used 
*** Number of reflection not used for refinement (free reflections)

**** Including double conformers

***** Number of atoms whose position was refined (atoms of active-state model)

$R_{\text {merge }}=\sum_{\mathrm{h}} \sum_{\mathrm{i}} \mid I(h, i)-\left\langle I(h)>\left|/ \sum_{\mathrm{h}} \sum_{\mathrm{i}}\right| I(h, i)\right| \times 100 \%$, where $I(h, i)$ is the intensity value of the $i$ th measurement of $h$ and $\langle I(h)>$ is the corresponding mean value of $h$ for all I measurements of $h$. The summation is over all measurements.

$R_{\text {work }}=\sum\left|F_{O}-F_{C}\right| /\left|\sum F_{O}\right| \times 100 \%$.

$R_{\text {free }}$ was calculated for $5 \%$ of observed reflections, omitted from the refinement and $R_{\text {work }}$ calculation, picked randomly within thin resolution shells. They are consistent between datasets. 


\section{Discussion}

We discuss the results in three different contexts. First, we compare the changes in the active state of uncomplexed NpSRII to those in NpSRII/NpHtrII complex. Second, we analyze the implications of the determined structure for understanding the proton pumping by NpSRII. And finally, we discuss the methodological aspects of observation of large conformational changes of proteins in crystals.

\section{Functionally important conformational changes in NpSRII}

The conformational changes observed for free NpSRII and NpSRII in complex with its transducer are quite similar in their nature (Figure 2). Major movements are observed for helices $\mathrm{F}$ and $\mathrm{G}$ which have been correlated with signal propagation from the receptor to the

transducer ${ }^{6,9,36,37}$. Additionally, an inward movement of the extracellular part of helix $\mathrm{C}$ is observed. Although these conformational changes are detected in both cases, in the case of NpSRII alone they have almost twice as large amplitudes (Figure 2). Smaller changes in the complexed NpSRII cannot be attributed to crystal packing, as it is looser in the crystals of NpSRII/NpHtrII, especially in the I212121 spacegroup (Ishchenko et al., to be published). Thus it means that the transducer opposes such structural rearrangements in the receptor. As this opposition leads to conformational changes in transducer itself, it constitutes a signal transfer.

In addition to the helix movements, we observe conformational changes in the E-F loop, which are not present in the NpSRII/NpHtrII complex. They may result from the crystal packing (E-F loops of adjacent proteins form a crystal contact), or may be a genuine conformational change in the active state of uncomplexed NpSRII. It has been shown by solid state NMR spectroscopy, fluorescence and EPR spectroscopy that the E-F loop is interacting tightly with the transducer ${ }^{38-40}$. However, it is clear that the E-F loop is not crucial 
for signal transduction, as NpSRII E-F loop deletion mutants as well as BR triple mutant, which has a small disordered E-F loop, are all capable of signaling ${ }^{20,41}$. The remaining question is whether the E-F loop enhances the signal transduction from NpSRII to NpHtrII or just serves for NpSRII stabilization.

So far we have discussed the large conformational changes between ground and active states of NpSRII. Unfortunately, due to the resolution of diffraction data, we are not able to observe neither the minute changes in the structure nor movements of the water molecules in the cytoplasmic channel. Nonetheless we are able to make a conclusion, that there are no changes in the uncomplexed NpSRII active state in the relative positions of the residues T204 and Y174 (Figure 3), which are proposed to be important for the signal generation in NpSRII/NpHtrII complex ${ }^{42,43}$. Probably, this reflects the spectroscopically observed absence of the alteration in T204-Y174 bonding in uncomplexed NpSRII ${ }^{44,45}$.

\section{Implications for NpSRII proton pumping}

Like BR, NpSRII is able to pump protons across the membrane, though less effectively. On formation of the NpSRII/NpHtrII complex this function is inhibited ${ }^{22,23}$. This inhibition may originate from kinetics alteration, from structural changes prohibiting the pumping, or from combination of these factors. Here, we analyze the implications of structural changes.

NpSRII active state transition results in disappearance of the water molecule, which makes a hydrogen bond to the retinal in the ground state (Figure 3). The same is observed in BR M state $^{46}$, as well as in NpSRII/NpHtrII complex $\mathrm{M}$ state ${ }^{9}$. There are also conformational changes of up to $1 \AA$ in the cytoplasmic channel, probably associated with the charge redistribution. Slight outward tilt of the G helix, combined with E-F loop changes, may facilitate the formation of water chain, which is believed to be needed for the retinal reprotonation and thus for completing the pumping cycle. These changes are not observed in 
the $\mathrm{M}$ state of complexed NpSRII, and may account for the NpHtrII proton pumping inhibition.

There are two ways in which NpHtrII may affect NpSRII dynamics. The first possibility is that NpHtrII may directly influence NpSRII conformational changes. The other possibility is as follows. The hydrophobic/hydrophilic boundary of NpSRII is uneven in the region of the helices $\mathrm{F}$ and $\mathrm{G}$, as seen from the surface potential distribution (Figure 1). Thus, the natural tendency of a lipid bilayer to be flat would result in energetic penalty for the NpSRII ground state. In the active state the mismatch is lowered, and thus the active state is preferred from the bilayer curvature energy point of view. NpHtrII shields the helices $\mathrm{F}$ and $\mathrm{G}$ from the lipid bilayer and thus reduces its influence on the NpSRII structure. In that case the energy of the active state is higher than in the absence of NpHtrII, and thus the conformational changes are lower.

\section{Observation of the large conformational changes of the protein in crystal}

As it was already mentioned, observation of the large conformational changes of the protein in crystal is complicated by the loss of the long range crystal order and the consequent diffraction loss. Therefore, special measures should be undertaken to obtain the tractable data. The parameters of the intermediate state accumulation should be thoroughly studied. The ideal loading procedure will result in not less than $40-50 \%$ of intermediate. At the same time, multiple transitions from the ground to intermediate state and back should be avoided, as the repetitive changes will cause more damage to the packing. Thus, the short loading time is preferred. Diffraction data should be measured from several crystals, to sample for those that diffract better, and for the consistency check of the observed changes. To obtain the best resolution possible, data should be treated with all the techniques available. In our case that meant utilization of the anisotropic data and construction of the full model with as many 
incorporated lipid molecules as possible. Finally, the intermediate state occupancy should be evaluated using crystallography techniques for each crystal, because often the crystals that seem to have better resolution, in fact contain not many protein molecules trapped in the intermediate state. 


\section{Materials and Methods}

\section{Protein preparation and crystallization}

Protein preparation and crystallization were performed as described previously ${ }^{47,48}$. The genes of NpSRII were cloned into a pET27bmod expression vector with a C-terminal 7x-His tag. Protein was expressed in E. coli strain BL21 (DE3), and purified as described ${ }^{47,48}$. After removal of imidazol by DEAE-chromatography, NpSRII-His was reconstituted into purple membrane lipids (protein to lipid ratio 1:35). After filtration, the reconstituted protein was pelleted by centrifugation at $100,000 \mathrm{x}$ g. For resolubilisation the samples were resuspended in a buffer containing $2 \%$ n-octyl- $\beta$-D-glucopyranoside $(\mathrm{OG})$ and shaken for $16 \mathrm{~h}$ at $4{ }^{\circ} \mathrm{C}$ in the dark. The resolubilized protein was isolated by centrifugation at 100,000 x g.

To the lipidic phase, formed from monooleoyl (NU-CHEK PREP, Inc., USA) the solubilized $20 \mathrm{mg} / \mathrm{ml}$ protein in crystallization buffer $(150 \mathrm{mM}, \mathrm{NaCl}, 25 \mathrm{mM} \mathrm{Na} / \mathrm{KPi}, \mathrm{pH} 5.1)$. Precipitant was $1 \mathrm{M}$ salt $\mathrm{Na} / \mathrm{KPi}$, pH 5.1 with $0.3 \mathrm{M}$ of trehalose. Crystals were grown at $22.5^{\circ} \mathrm{C}$.

It should be noted that the addition of trehalose improved the quality of the grown NpSRII crystals. It cannot be excluded that the improvement is due to the „trapped“ dynamic protein conformations ${ }^{49}$. Nevertheless, two other possible sources of the influence of trehalose on the quality of in meso grown membrane protein crystals - the improvement of cryoprotection and direct influence on crystallization matrix - should be mentioned as well. The ongoing study will help to understand better the observed effect.

\section{Trapping of intermediates}

For the loading of the intermediate state the crystals were illuminated with an an argonkrypton ion laser with wavelength $488 \mathrm{~nm}$ (Omnichrome). Cryostream was blocked was 
blocked for $2 \mathrm{~s}$. The illumination was turned off one second after recooling started. X-ray data were collected in darkness.

\section{Spectroscopic characterization of the crystals}

Visual spectra of single crystals were measured with an IR-VIS spectrophotometer described elsewhere ${ }^{50}$. Spectra were recorded from single crystals in the nitrogen cryostream under conditions identical to those used for X-ray measurements. Data were fitted by skewed gaussians with modified parameters from Chizhov et al. ${ }^{51}$

\section{Acquisition and treatment of diffraction data}

X-ray diffraction data (wavelength $0.934 \AA$ ) for the ground and the active states were collected at beamlines ID14-1 and ID23-1 at of the European Synchrotron Radiation Facility (ESRF), Grenoble, France correspondingly. Image acquisition time was $10 \mathrm{~s}$, oscillation range $0.9^{\circ}$, and a total of 105 images were collected in each dataset. Diffraction patterns were processed with MOSFLM/SCALA $\left(\mathrm{CCP}^{52}\right)$. Anisotropic correction was performed via anisotropy server ${ }^{53}$. Crystallographic parameters and refinement statistics are summarized in Table 1.

\section{Crystallographic refinement}

For ground state structure determination PDB ID $\underline{\mathbf{1 H 6 8}}$ model was iteratively refined using REFMAC $5^{54}$ and $\operatorname{Coot}^{55}$. For usage in signalling state structure determination, the resulting ground state structure was minimized in $\mathrm{CNS}^{56}$, in order to equilibrate the model with $\mathrm{CNS}$ parameter set. q-weighted difference density maps built with CNS were used for preliminary analysis of the structural changes ${ }^{57}$. Most differences were located in the retinal-binding pocket and along the $\mathrm{F}$ and $\mathrm{G}$ helices. Isomerisation and deviation of the retinal out of the F- 
helix, as well as displacement of W171 are clearly seen for all datasets. Changes for different crystals were similar.

The intermediate state refinement was conducted as follows. First, the ground state model was subjected to rigid body refinement. Then the part of it, corresponding to the ground state (occupancy 55\%) was fixed, and the other part, corresponding to the intermediate (occupancy $45 \%$ ) was subjected to simulated annealing. For the annealed part of the model, a less ordered loop and terminal residues (1-2, 28-32 and 217-219) were removed, along with surface water molecules and small lipid fragments. Internal water molecules, expected to be present but not clearly seen at present resolution, were harmonically restrained to positions in the groundstate structure. After simulated annealing procedure conformations of some residues were manually changed towards ideal geometry.

\section{Accession codes}

Coordinates and structure factors have been deposited to the PDB under the accession codes

\section{QAP and 3QDC.}

\section{Acknowledgments}

We would like to thank the Structural Biology Group of ESRF. We are also thankful to C. Baeken for the help with NpSRII preparation.

The work was supported by the program "Chaires d'excellence" edition 2008 of ANR France, CEA(IBS) - HGF(FZJ) STC 5.1 specific agreement and the MC grant for training and career development of researchers (Marie Curie, FP7-PEOPLE-2007-1-1-ITN, project SBMPs). The work was done in the framework of Russian State Contracts № 02.740.11.0299, № 02.740.11.5010, №P974 in the framework of activity 1.2.2 and №P211 in the framework of 
activity 1.3.2 of the Federal Target Program «Scientific and academic research cadres of innovative Russia» for 2009-2013 years.

Partial financial support from ONEXIM group is gratefully aknowledged.

\section{Supporting materials}

Supporting materials describing the intermediate state occupancy determination by spectroscopic and crystallographic methods are available. 


\section{References}

1. Sharma, A.K., Spudich, J.L. \& Doolittle, W.F. Microbial rhodopsins: functional versatility and genetic mobility. Trends in Microbiology 14, 463-469 (2006).

2. Klare, J., Chizhov, I. \& Engelhard, M. Microbial Rhodopsins: Scaffolds for Ion Pumps, Channels, and Sensors. Bioenergetics: Energy conservation and conversion (eds. G. Schäfer, H.S. Penefsky) 73-122 (2008).

3. Zhang, F. et al. Multimodal fast optical interrogation of neural circuitry. Nature 446, 633639 (2007).

4. Sasaki, J. \& Spudich, J.L. Signal Transfer in Haloarchaeal Sensory Rhodopsin Transducer Complexes. Photochemistry and Photobiology 84, 863-868 (2008).

5. Gordeliy, V.I. et al. Molecular basis of transmembrane signalling by sensory rhodopsin II-transducer complex. Nature 419, 484-487 (2002).

6. Wegener, A.A., Klare, J.P., Engelhard, M. \& Steinhoff, H.J. Structural insights into the early steps of receptor-transducer signal transfer in archaeal phototaxis. EMBO J 20, 5312-5319 (2001).

7. Wegener, A.-A., Chizhov, I., Engelhard, M. \& Steinhoff, H.-J. Time-resolved detection of transient movement of helix $\mathrm{F}$ in spin-labelled pharaonis sensory rhodopsin II. Journal of Molecular Biology 301, 881-891 (2000).

8. Bordignon, E. et al. Analysis of light-induced conformational changes of Natronomonas pharaonis sensory rhodopsin II by time resolved electron paramagnetic resonance spectroscopy. Photochemistry and photobiology 83, 263-272 (2007).

9. Moukhametzianov, R. et al. Development of the signal in sensory rhodopsin and its transfer to the cognate transducer. Nature 440, 115-119 (2006).

10. Hazelbauer, G.L., Falke, J.J. \& Parkinson, J.S. Bacterial chemoreceptors: highperformance signaling in networked arrays. Trends in Biochemical Sciences 33, 9-19 (2008).

11. Luecke, H., Schobert, B., Lanyi, J.K., Spudich, E.N. \& Spudich, J.L. Crystal Structure of Sensory Rhodopsin II at 2.4 Angstroms: Insights into Color Tuning and Transducer Interaction. Science 293, 1499-1503 (2001).

12. Royant, A. et al. X-ray structure of sensory rhodopsin II at 2.1- $\AA$ resolution. Proceedings of the National Academy of Sciences of the United States of America 98, 10131-10136 (2001).

13. Reshetnyak, A.B. et al. Comparative analysis of sensory rhodopsin II structures in complex with a transducer and without it. Journal of Surface Investigation: X-ray, Synchrotron and Neutron Techniques 2, 894-899 (2008).

14. Gautier, A., Mott, H.R., Bostock, M.J., Kirkpatrick, J.P. \& Nietlispach, D. Structure determination of the seven-helix transmembrane receptor sensory rhodopsin II by solution NMR spectroscopy. Nat Struct Mol Biol 17, 768-774 (2010).

15. Luecke, H., Schobert, B., Richter, H.T., Cartailler, J.P. \& Lanyi, J.K. Structure of bacteriorhodopsin at $1.55 \AA$ resolution. Journal of molecular biology 291, 899-911 (1999).

16. Sasaki, J. \& Spudich, J.L. Proton Circulation During the Photocycle of Sensory Rhodopsin II. Biophysical Journal 77, 2145-2152 (1999).

17. Bogomolni, R.A. et al. Removal of transducer HtrI allows electrogenic proton translocation by sensory rhodopsin I. Proceedings of the National Academy of Sciences of the United States of America 91, 10188 -10192 (1994). 
18. Iwamoto, M., Shimono, K., Sumi, M., Koyama, K. \& Kamo, N. Light-Induced Proton Uptake and Release of pharaonis Phoborhodopsin Detected by a Photoelectrochemical Cell. The Journal of Physical Chemistry B 103, 10311-10315 (1999).

19. Schmies, G. et al. Sensory rhodopsin II from the haloalkaliphilic natronobacterium pharaonis: light-activated proton transfer reactions. Biophys J 78, 967-976 (2000).

20. Sudo, Y. \& Spudich, J.L. Three strategically placed hydrogen-bonding residues convert a proton pump into a sensory receptor. Proceedings of the National Academy of Sciences 103, 16129-16134 (2006).

21. Klare, J.P. et al. Probing the proton channel and the retinal binding site of Natronobacterium pharaonis sensory rhodopsin II. Biophys J 82, 2156-2164 (2002).

22. Schmies, G., Engelhard, M., Wood, P.G., Nagel, G. \& Bamberg, E. Electrophysiological characterization of specific interactions between bacterial sensory rhodopsins and their transducers. Proc. Natl. Acad. Sci. U.S.A 98, 1555-1559 (2001).

23. Sudo, Y., Iwamoto, M., Shimono, K., Sumi, M. \& Kamo, N. Photo-induced proton transport of pharaonis phoborhodopsin (sensory rhodopsin II) is ceased by association with the transducer. Biophys. J 80, 916-922 (2001).

24. Sasaki, J. \& Spudich, J.L. Proton transport by sensory rhodopsins and its modulation by transducer-binding. Biochimica et Biophysica Acta (BBA) - Bioenergetics 1460, 230-239 (2000).

25. Edman, K. et al. Early structural rearrangements in the photocycle of an integral membrane sensory receptor. Structure 10, 473-482 (2002).

26. Ruprecht, J.J., Mielke, T., Vogel, R., Villa, C. \& Schertler, G.F. Electron crystallography reveals the structure of metarhodopsin I. EMBO J 23, 3609-3620 (2004).

27. Choe, H.-W. et al. Crystal structure of metarhodopsin II. Nature 471, 651-655 (2011).

28. Taniguchi, Y., Ikehara, T., Kamo, N., Yamasaki, H. \& Toyoshima, Y. Dynamics of Light-Induced Conformational Changes of the Phoborhodopsin/Transducer Complex Formed in the n-Dodecyl $\beta$-d-Maltoside Micelle. Biochemistry 46, 5349-5357 (2007).

29. Yan, B., Takahashi, T., Johnson, R. \& Spudich, J.L. Identification of signaling states of a sensory receptor by modulation of lifetimes of stimulus-induced conformations: the case of sensory rhodopsin II. Biochemistry 30, 10686-10692 (1991).

30. Hein, M., Wegener, A.A., Engelhard, M. \& Siebert, F. Time-Resolved FTIR Studies of Sensory Rhodopsin II (NpSRII) from Natronobacterium pharaonis: Implications for Proton Transport and Receptor Activation. Biophysical Journal 84, 1208-1217 (2003).

31. Bergo, V., Spudich, E.N., Spudich, J.L. \& Rothschild, K.J. Conformational Changes Detected in a Sensory Rhodopsin II-Transducer Complex. Journal of Biological Chemistry 278, 36556 -36562 (2003).

32. Furutani, Y. et al. FTIR Spectroscopy of the O Photointermediate in pharaonis Phoborhodopsin. Biochemistry 43, 5204-5212 (2004).

33. Inoue, K., Sasaki, J., Spudich, J.L. \& Terazima, M. Laser-Induced Transient Grating Analysis of Dynamics of Interaction between Sensory Rhodopsin II D75N and the HtrII Transducer. Biophysical Journal 92, 2028-2040 (2007).

34. Subramaniam, S.B.P., Tittor, J.O.D. \& Lanyi, J.H.R. Protein conformational changes in the bacteriorhodopsin photocycle. Journal of Molecular Biology 287, (1999).

35. Sass, H.J. et al. Structural alterations for proton translocation in the M state of wild-type bacteriorhodopsin. Nature 406, 649-653 (2000).

36. Bergo, V.B., Spudich, E.N., Rothschild, K.J. \& Spudich, J.L. Photoactivation Perturbs the Membrane-embedded Contacts between Sensory Rhodopsin II and Its Transducer. Journal of Biological Chemistry 280, 28365 -28369 (2005).

37. Yoshida, H., Sudo, Y., Shimono, K., Iwamoto, M. \& Kamo, N. Transient movement of helix $\mathrm{F}$ revealed by photo-induced inactivation by reaction of a bulky $\mathrm{SH}$-reagent to 
cysteine-introduced pharaonis phoborhodopsin (sensory rhodopsin II). Photochem. Photobiol. Sci 3, 537-542 (2004).

38. Bordignon, E. et al. Structural Analysis of a HAMP Domain. Journal of Biological Chemistry 280, 38767 -38775 (2005).

39. Etzkorn, M. et al. Complex Formation and Light Activation in Membrane-Embedded Sensory Rhodopsin II as Seen by Solid-State NMR Spectroscopy. Structure 18, 293-300 (2010).

40. Yang, C.-S., Sineshchekov, O., Spudich, E.N. \& Spudich, J.L. The Cytoplasmic Membrane-proximal Domain of the HtrII Transducer Interacts with the E-F Loop of Photoactivated Natronomonas pharaonis Sensory Rhodopsin II. J. Biol. Chem. 279, 42970-42976 (2004).

41. Sasaki, J., Nara, T., Spudich, E.N. \& Spudich, J.L. Constitutive activity in chimeras and deletions localize sensory rhodopsin II/HtrII signal relay to the membrane-inserted domain. Molecular Microbiology 66, 1321-1330 (2007).

42. Sudo, Y., Furutani, Y., Kandori, H. \& Spudich, J.L. Functional importance of the interhelical hydrogen bond between Thr204 and Tyr174 of sensory rhodopsin II and its alteration during the signaling process. Journal of Biological Chemistry 281, 34239 (2006).

43. Ito, M. et al. Steric Constraint in the Primary Photoproduct of Sensory Rhodopsin II Is a Prerequisite for Light-Signal Transfer to HtrII†. Biochemistry 47, 6208-6215 (2008).

44. Furutani, Y., Sudo, Y., Kamo, N. \& Kandori, H. FTIR Spectroscopy of the Complex between pharaonis Phoborhodopsin and Its Transducer Protein $\uparrow$. Biochemistry 42, 48374842 (2003).

45. Sudo, Y., Furutani, Y., Shimono, K., Kamo, N. \& Kandori, H. Hydrogen Bonding Alteration of Thr-204 in the Complex between pharaonis Phoborhodopsin and Its Transducer Protein $\dagger$. Biochemistry 42, 14166-14172 (2003).

46. Lanyi, J.K. Proton transfers in the bacteriorhodopsin photocycle. Biochimica et Biophysica Acta (BBA) - Bioenergetics 1757, 1012-1018 (2006).

47. Hohenfeld, I.P., Wegener, A.A. \& Engelhard, M. Purification of histidine tagged bacteriorhodopsin, pharaonis halorhodopsin and pharaonis sensory rhodopsin II functionally expressed in Escherichia coli. FEBS Letters 442, 198-202 (1999).

48. Shimono, K., Iwamoto, M., Sumi, M. \& Kamo, N. Functional expression of pharaonis phoborhodopsin in Eschericha coli. FEBS Letters 420, 54-56 (1997).

49. Cordone, L., Ferrand, M., Vitrano, E. \& Zaccai, G. Harmonic Behavior of TrehaloseCoated Carbon-Monoxy-Myoglobin at High Temperature. Biophysical Journal 76, 10431047 (1999).

50. Gordeliy, V.I., Schlesinger, R., Efremov, R., Büldt, G. \& Heberle, J. Crystallization in Lipidic Cubic Phases. Membrane Protein Protocols: Expression, Purification, and Characterization (ed. Selinsky, B) (2003).

51. Chizhov, I. et al. The photophobic receptor from Natronobacterium pharaonis: temperature and $\mathrm{pH}$ dependencies of the photocycle of sensory rhodopsin II. Biophys. $J$ 75, 999-1009 (1998).

52. Collaborative Computational Project Number 4 The CCP4 suite: programs for protein crystallography. Acta Crystallogr D Biol Crystallogr 50, 760-763 (1994).

53. Strong, M. et al. Toward the structural genomics of complexes: crystal structure of a PE/PPE protein complex from Mycobacterium tuberculosis. Proceedings of the National Academy of Sciences 103, 8060 (2006).

54. Murshudov, G.N., Vagin, A.A. \& Dodson, E.J. Refinement of macromolecular structures by the maximum-likelihood method. Acta Crystallographica Section D: Biological Crystallography 53, 240-255 (1997). 
55. Emsley, P. \& Cowtan, K. Coot: model-building tools for molecular graphics. Acta Crystallographica Section D: Biological Crystallography 60, 2126-2132 (2004).

56. Brunger, A.T. et al. Crystallography \& NMR system: a new software suite for macromolecular structure determination. Acta Crystallographica Section D: Biological Crystallography 54, 905-921 (1998).

57. Ursby, T. \& Bourgeois, D. Improved Estimation of Structure-Factor Difference Amplitudes from Poorly Accurate Data. Acta Crystallogr A Found Crystallogr 53, 564575 (1997).

58. Baker, N.A., Sept, D., Joseph, S., Holst, M.J. \& McCammon, J.A. Electrostatics of nanosystems: Application to microtubules and the ribosome. Proceedings of the National Academy of Sciences of the United States of America 98, 10037 -10041 (2001). 

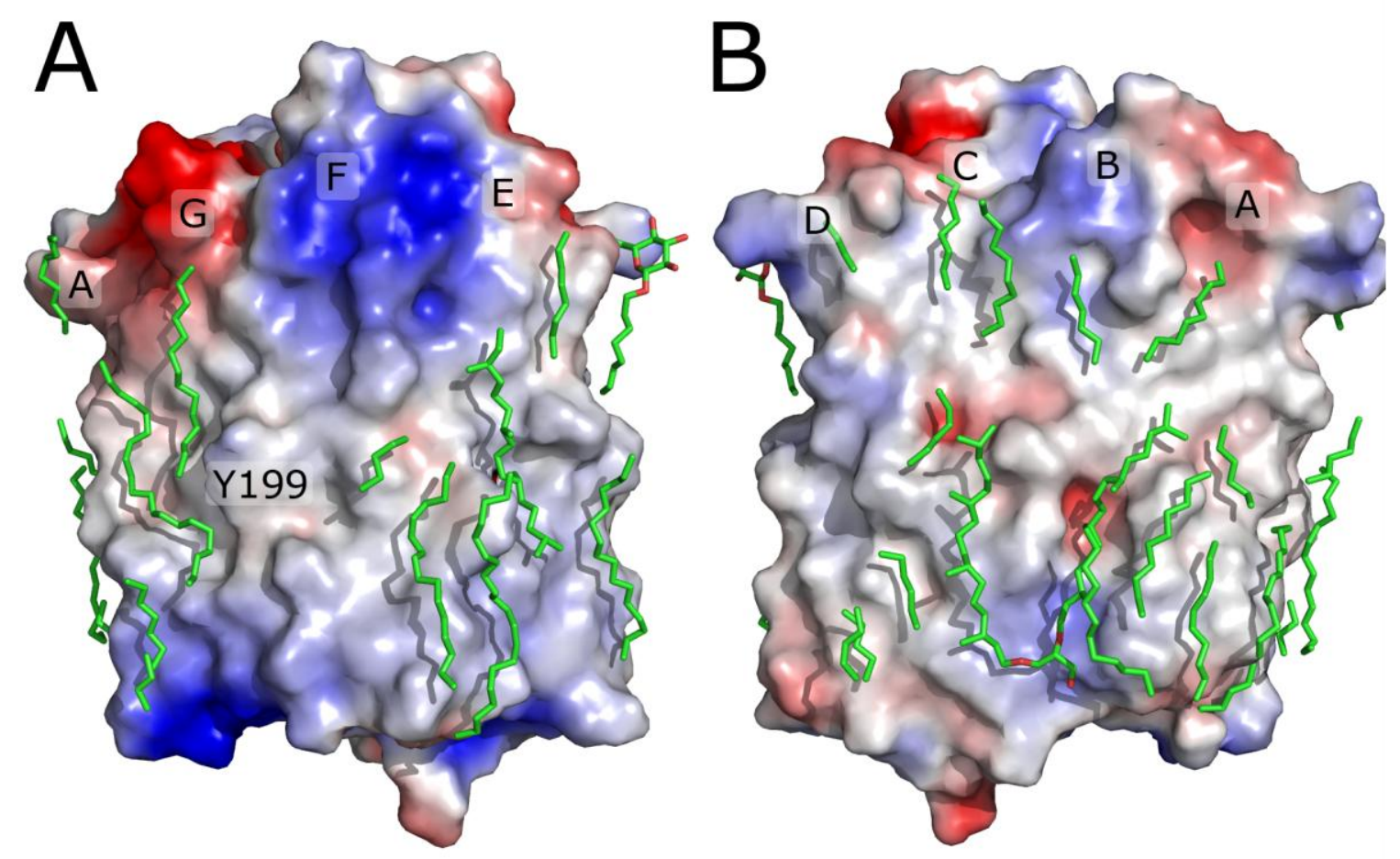

Figure 1. Lipid tails in the ground state model of NpSRII. Lipid tails are shown in green sticks representations. Surface potential was calculated using $\mathrm{ABPS}^{58}$. Blue color corresponds to potential value of $+3 \mathrm{kT} / \mathrm{e}$ and higher, and red color to $-3 \mathrm{kT} / \mathrm{e}$ and lower. Lipid bilayer was not included in the calculation. NpSRII helices are marked by corresponding letters. Tyrosine Y199 and the region of positive potential near the helix F correspong to the place of NpHtrII transducer binding in NpSRII-NpHtrII complex. 

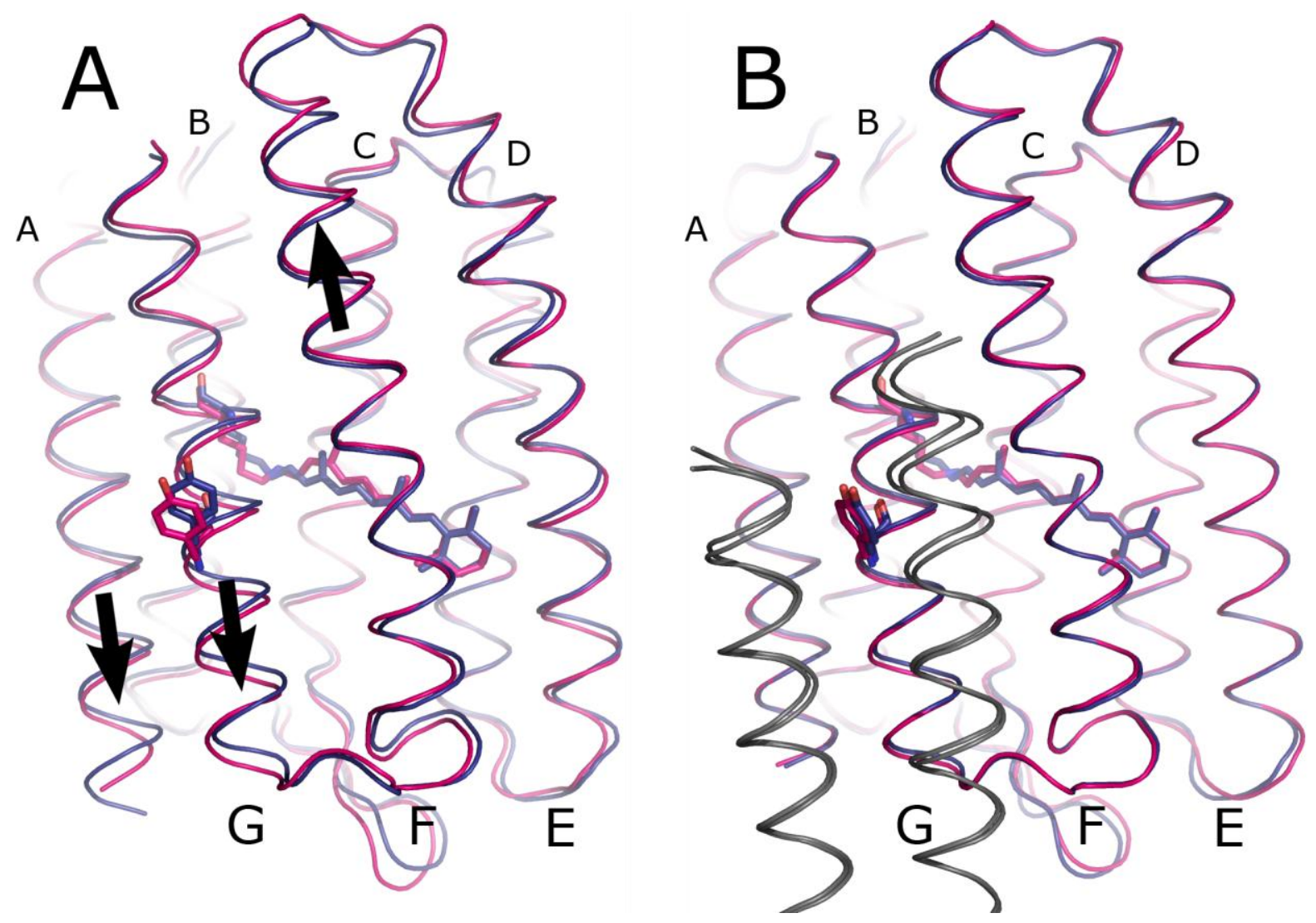

Figure 2. Overall structural rearrangements in NpSRII upon transition to the active state. A) Structural rearrangements in uncomplexed NpSRII. B) Structural rearrangements in NpSRII in complex with $\mathrm{NpHtrII}^{9}$ (PDB ID $\underline{\mathbf{2 F 9 5}}$ ). The ground state is shown in blue and the active state is in magenta. NpHtrII in B) is shown in grey for both states. Lysine 205, retinal and tyrosine 199 are shown as a ball-and-stick models. NpSRII helices are marked by corresponding letters (the larger letters correspond to the front helices, and the smaller letters correspond to the back helices). Arrows show movements of the corresponding helices. Alignment is done by the NpSRII backbone atoms. 


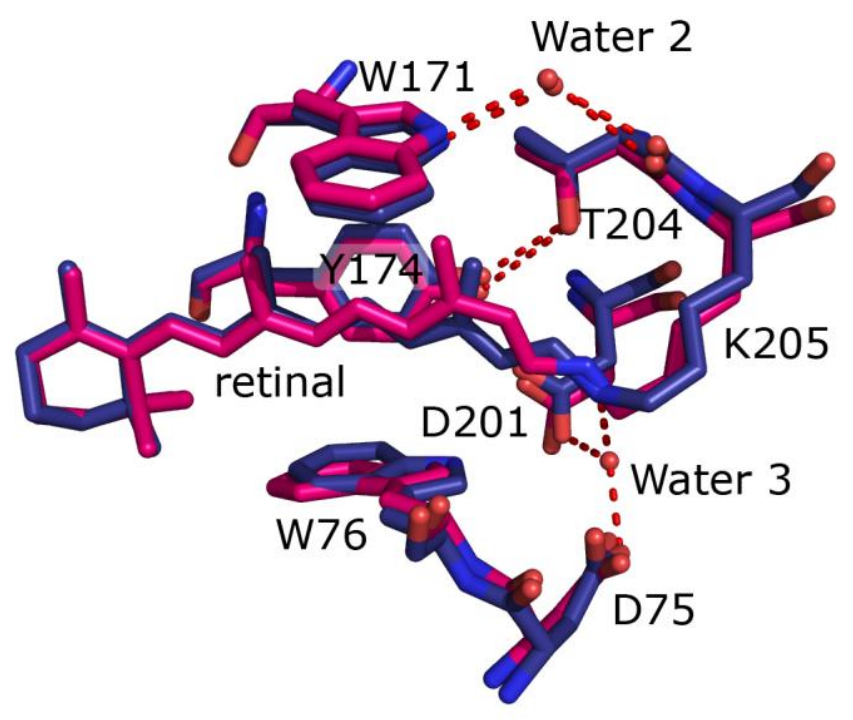

Figure 3. Comparison of the retinal environment in the NpSRII ground state (in blue) and active state (in magenta) structures. Note that the active state is a mix of the $\mathrm{M}$ and $\mathrm{O}$ states, and retinal is shown only as it would be in the $\mathrm{M}$ state in 13-cis conformation. Positions of other residues represent the averages of those in the $\mathrm{M}$ and $\mathrm{O}$ states and reflect the general conformational changes in this region. Hydrogen bonds are shown by dashed lines. Water molecule 3 disappears in the active state. 